An Alternate Scale Representation of Atmospheric Energy Spectra

\author{
By \\ Ferdinand Baer
}

Department of Atmospheric Science

Colorado State University

Fort Collins, Colorado

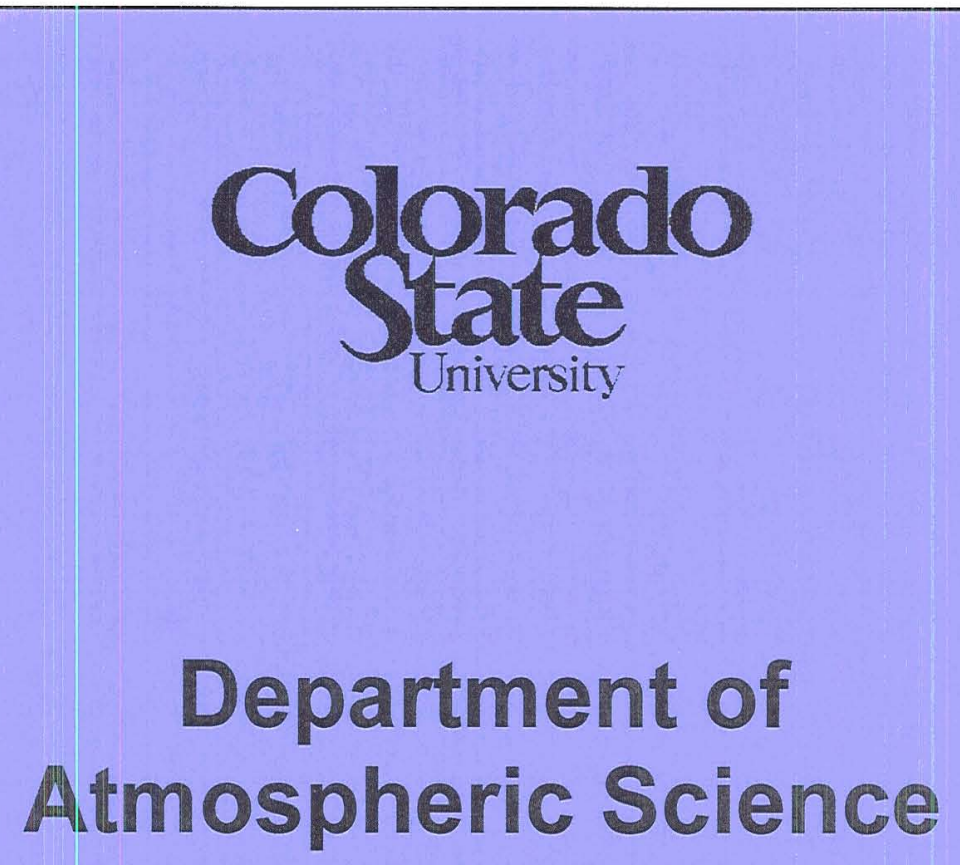

Paper No. 175 


\title{
An Alternate Scale Representation of Atmospheric Energy Spectra
}

\author{
FERDIAAND BAER ${ }^{\mathrm{i}}$ \\ Dept. of Amospheric Science, Colorado Stale Linicrsity, Furt Collins
}

Manuscript received 7 September 1971)

\section{ABSTRACT}

\begin{abstract}
Although it is known that all spatial scales are nonlinearly interrelated in any prediction model of the atmosphere, truncation demands a limit to scale resolution. One is therefore compelled to parameterize sub-resolution scales, hryefully in such a mamner that the describe observed statistics. Such statistics have been shown frequently as energy spectra of synoptic scales in terms of the planetary wavenumber. An alternate representation is the presentation of the energy in terms of the degree of a Legendre polynomial expansion; this representation may be more advantageous insofar as it presents a two-dimensional sjectral index Arguments are presented which indeed suggest the appropriateness of the index. Two months of atmospheric wind dat a at five pressure levels and on a hemispheric grid were analyzed to establish energy spectra. The spectra are described both as a function of time and as a function of wavenumber for time averages. Using a tive-level linear baroclinic model, stability characteristics for each wave component for the observed zonal and vertical profiles were established. Based on these results, the energy data were fit lorarithmically by least squares to the wavenumber both planetary wavenumber and Legendre polynomial degree). Energy slopes show values close to -3 when utilizing the two-dimensional index in the non-baroclinically forceil scale range. These results sugrgest the use of this index in studying scale parameterization.
\end{abstract}

\section{Introduction}

The question of atmospheric predictability has attracted the attention of meteorologists with increasing urgency over the past few years, concomitant with the development of model sophistication and applicable computer technology. Indeed, the proposals of GARP relating to prediction periods have catapulted the question into international prominence. Nevertheless, no definitive answers have yet been forthcoming although valuable contributions have been made on both the positive and negative sides, notably by Robinson (1967), Lorenz (1969), Smagorinsky (1969) and Leith (1971). These arguments range from the possibility of satisfactory long-range prediction with improved computing power to absolute limits on prediction due to error propayation.

Although it is not the purpose of this paper to speculate on the prospects of atmospheric predictability, we do wish to clarify at least one factor which is clearly involved and suggest a representation which will lead to a better understanding of this factor and hopefully assist in the subsequent evaluation of the prediction process. It is common knowledge that becuse the atmosphere is a nonlinear system, all scales a.te interactive. However, despite superior computing power, no model calculation can hope to resolve all scales. One is consequently led to the unhappy but inevitable altemative of parameterizing a portion of

Present affiliation: Department of Meteorology and Oceanayritphy, The Cniversity of Michigan, Ann Arbor. the scale domain (usually the shorter scales), a procedure sometimes termed closure, and recently considered by Leith (1968).

If then-and we assert as a necessity-the shorter scales must be parameterized so that the longer scales may be satisfactorily predicted for a specified time, we must first have observational knowledge concerning some statistical properties of the shorter scales. Finding such statistics by random search clearly would be futile. Fortunately, recent advances in two-dimensional turbulence theory (Kraichnan, 1967) suggest a possibly meaningful statistic. This theory states that in a twodimensional viscous fluid with an energy source confined to a narrow scale region, the energy distribution in scales shorter than the forcing scale will decrease logarithmically with the two-dimensional scale index with a slope of -3 . Should the atmosphere manifest two-dimensional behavior in part of the scale range, by analogy with turbulence theory, the energy distribution in this scale range can be assessed from observation and may yield just the desired statistic sought for closure purposes. The atmosphere is certainly a three-dimensional fluid. However, baroclinic stability theory suggests that the predominant three-dimensionality tends to assert itself in a limited scale range (principally in planetary waves $6-10$ ) and that in shorter scales there is little vertical overturning. Thus, one might speculate that the scales shorter than planetary waves 10 behave in a quasi two-dimensional sense and, by analogy with Kraichnan's theory, have an energy source in the range 
CARTESIAN DOMAIN

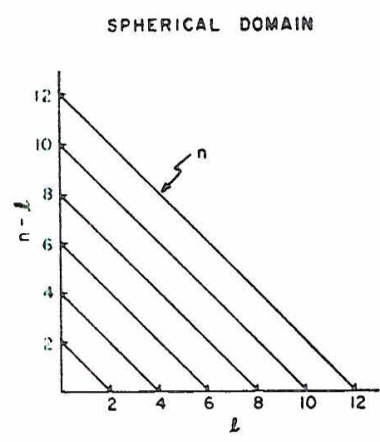

FIg. 1. Distribution of the two-dinensional scale index in terms of the two one-dimensional indices for both Cartesian and spherical domains.

of waves 6-10. The observational data analyses of Wiin-Nielsen (1967) and Julian et al. (1970) tend to substantiate a -3 energy distribution in the planetary wave distribution.

Despite these apparently favorable observations, they are based on a one-dimensional analysis and are therefore not comparable to the expectations from two-dimensional theory; consequently, they also cannot provide a satisfactory closure condition. We must find a two-dimensional representation for the atmosphere which yields observations corresponding to two-dimensional turbulence theory. Such a representation has been used by Lilly (1969) to calculate the energy distribution vs two-dimensional scale in a simple turbulence model with remarkable success, i.e., he calculated the anticipated -3 slope. The geometry utilized by Lilly in his model is unfortunately inapplicable to the largest atmospheric scales because of the earth's curvature.

It is the purpose of this study to present a twodimensional scale representation applicable to variables in atmospheric surfaces (pressure or geopotential) and to describe observed energy distributions in terms of this scale. The appropriateness of the scale representation will be assessed, the region of two-dimensionality with regard to this scale will be established, and the -3 distribution of energy in terms of the scale index will be highlighted. Based on the successful comparison of observed energy distributions with twodimensional turbulence theory in terms of our proposed two-dimensional scale representation, that representation should lead to a suitable parameterization of shorter scales with known statistics and thereby advance our understanding of the predictability question.

\section{An appropriate two-dimensional index}

To establish an index which characterizes scales in a two-dimensional surface and which will permit an expansion of variables in those scales, let us consider a two-dimensional differential operator as it operates on a normalized function. Such an operator, say the Laplacian $\left(\nabla^{2}\right)$, would by simple scale analysis have the dimensions

$$
\nabla^{2} \sim\left(\frac{1}{\Delta s}\right)^{2} \sim\left(\frac{m}{s}\right)^{2},
$$

where $s^{2}$ is the overall area of the region and $m$ is an index (the sought after two-dimensional index) representing the number of subregions $(\Delta s)^{2}$ in the total domain; i.e., $(\Delta s)^{2}=(s / m)^{2}$.

Let us now apply this operator to a rectangular region described in a Cartesian representation wherein the function to be operated on is periodic in both dimensions. The characteristic functions with these properties are clearly given as

$$
f(\boldsymbol{r})=\exp (i \mathbb{k} \cdot \mathbf{r})
$$

where $\mathbf{k}=\mathbf{k}_{x} \mathbf{i}+k_{y} \mathbf{j}$ and $\mathbf{r}$ is the radius vector in the surface. By application of the Laplace operator

$$
\nabla^{2} f=-k^{2} f, \text { where } k^{2}=k_{x}^{2}+k_{y}^{2},
$$

and we see from $m^{2}=k^{2} s^{2}$ that the appropriate twodimensional index for this domain is $k^{2}$. Indeed this is the index which was used by Lilly (1969) in his numerical simulation of two-dimensional turbulence. The distribution of $k^{2}$ as it depends on $k_{x}$ and $k_{y}$ is depicted graphically on the left-hand side of Fig. 1. Note that $k_{x}$ and $k_{y}$ represent one-dimensional indices of scale in the $x$ and $y$ directions, respectively.

For problems dealing with representation in a spherical surface (obviously applicable to the earth's atmosphere), the appropriate characteristic functions are the solid spherical harmonics

$$
Y_{\alpha}(\lambda, \mu)=\exp \left(i l_{\alpha} \lambda\right) P_{\alpha}(\mu)
$$

where $\alpha \equiv n_{\alpha}+i l_{\alpha}$ is a complex wave vector describing the two one-dimensional scales; i.e., $l_{\alpha}$ represents the planetary (longitudinal) scale index and $n_{\alpha}-l_{\alpha}$ the ordinal (latitudinal) index, defining the number of zeros between the poles. The longitude is represented by $\lambda, \mu$ describes the sine of latitude, and the associated Legendre polynomials $\left(P_{\alpha}\right)$ are polynomials in $\mu$ of order $n_{\alpha}$. Application of the Laplace operator to these functions leads to the well-known characteristic equation

$$
\nabla^{2} Y_{\alpha}=-n_{\alpha}\left(n_{\alpha}+1\right) Y_{\alpha} .
$$

The appropriate two-dimensional scale index is here seen to be $m^{2}=n_{\alpha}\left(n_{\alpha}+1\right) s^{2}$, which depends not only on $n_{\alpha}$ alone, but is linearly proportional to $n_{\alpha}$ for indices not too close to unity. It is this index then, the order of the Legendre polynomial, which we wish to explore as a possible alternative scale index for twodimensional representation in a spherical surface. By suggesting our scale index as an alternative, we imply that atmospheric variables have traditionally been described by the one-dimensional index $l_{\alpha}$ alone and hemispheric averages have been extracted by integration over latitude of the variables as a function of $l_{\alpha}$ and $\mu$. This procedure may be observed in the analyses of Saltzman : Julian et al. (

The distrib one-dimensior cally on the larity of dist index of the given value Cartesian an from Fig. 1. for each valuı of all ampliti: of constant is discuss the $\mathrm{k}$ detail subsec fived $k$ in th maly not be We therefore st ructures for type projectis be displayed. how the cell: tude $\left(l_{\alpha}\right)$ and cell configura 
is an repretotal

agular herein both these

in the

e twothis is is nue. The spicted te that if scale

in a earth's nctions scribing $n$ ts the $-l_{\alpha}$ the aber of esented sociated in $\mu$ of to these quation

is here lot only $n_{\alpha}$ for hen, the wish to for twoace. $B y$ e imply been deone and integraion of $l$. analyses
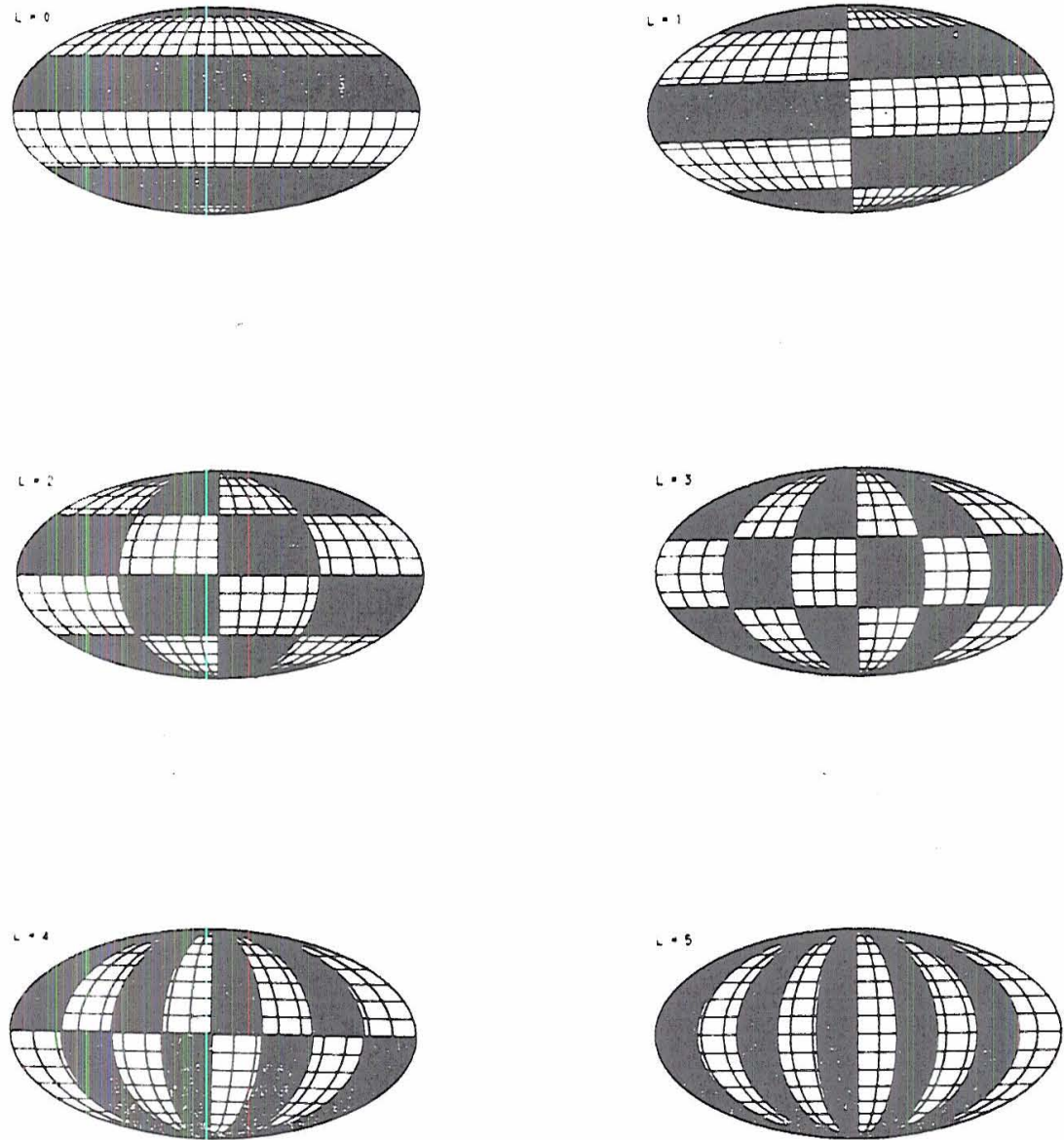

Vig. 2 Frample" of ditferent cell'contionations all having the same two-dimensional index, in this case: $n=5$. The cells are detined by their nodal lines and are presented on a Mollweide-type projection.

of Saltzman and Fleisher (1962), Wiin-Nielsen (1967), Julian et al. (1970) and others.

The distribution of the index $n_{\alpha}$ in terms of the two one-dimensional indices $l_{x}, n_{x}-l_{\alpha}$ is described graphicalls on the right-hand side of Fig. 1. Note the similarity of distribution between this index and the $k^{2}$ index of the Cartesian plane. The cells included in a given value of the two-dimensional index in both Cartesian and spherical domains also may be seen from Fig. 1. The total amplitude of a given variable for atch value of an index would require the summation of all amplitudes at allowed intersections along a curve of constant index value $\left(k\right.$ or $\left.n_{\alpha}\right)$---see Fig. 1. We shall discuss the kinetic energy as such a variable in more detail subsequently. Although the structure of cells for fixes $k$ in the Cartesian domain is well known, such may not be the case for cells in a spherical surface. $W_{e}$ therefore present as an example the allowed cell structures for $n_{x x}=5$ in Fig. 2. By use of the Mollweidetype projection (Steers, 1965 ), all $360^{\circ}$ of longitude may be displaved. It should be evident from this example how the cell structure depends upon the zeros in longitude $\left(l_{x}\right)$ and the zeros in latitude $\left(n_{x}-l_{\alpha}\right)$. The limit in cell configurations is based on the condition for Legendre polynomials that $n_{\alpha} \geqslant l_{\alpha x}$. Finally, with regard to the conventional representation in terms of $l_{a x}$ alone, only the structure for $l=5$ in Fig. 2 would come under consideration, i.e., no wave structure with latitude is generally considered.

The argument for selecting the two-dimensional index presented above is based essentially on dimensional analysis. It is of some interest to note, however, that the wave components of a given scale (e.g., Fig. 2) will not interact nonlinearly with one another in a twodimensional turbulent flow, thus suggesting their scale independence and supporting our choice of index. As an extension of the results of Neamtan (1946) and Platzman (1960) indicating the existence of an exact solution to the nonlinear barotropic vorticity equation subject to suitable wave truncation, the non-interaction of wave components with fixed scale (two dimensional) may be elucidated. Considering the flow to be represented by a streamfunction, and letting that streamfunction be separated into a zonal part $(\bar{\psi})$ comprising an arbitrary number of zonal components in an expansion of zonal harmonics and a wave part $\left(\psi^{\prime}\right)$ comprising an arbitrary number of planetary wave components of fixed ordinal index $(n)$-this latter choice 
based on our desire to investigate the interaction of wave components of given scale-the vorticity equation may be written in a spherical surface as

$$
\begin{array}{r}
\frac{\partial \nabla^{\prime 2}}{\partial t}\left(\bar{\psi}+\psi^{\prime}\right)=J\left(\nabla^{2} \bar{\psi}, \bar{\psi}\right)+J\left(\nabla^{2} \psi^{\prime}, \psi^{\prime}\right)+J\left(\nabla^{2} \psi^{\prime}, \bar{\psi}\right) \\
+J\left(\nabla^{\prime} \bar{\psi}, \psi^{\prime}\right)+J\left(f, \bar{\psi}+\psi^{\prime}\right)+L\left(\psi^{\prime}\right),
\end{array}
$$

where

$$
\begin{aligned}
\bar{\psi}(\mu, t) & =\sum_{m} \psi_{m}(t) P_{m}(\mu), \quad l=0 \\
\psi^{\prime}(\lambda, \mu, t) & =\sum_{l_{\alpha}} \psi_{\alpha}(t) Y_{\alpha}(\lambda, \mu) \\
& \times\left\{\alpha=n+i l_{\alpha}, n \text { fixed, } l_{\alpha} \neq 0\right\}
\end{aligned}
$$

In (3) the usual notation applies; $J$ is the Jacobian operator, $f$ the Coriolis parameter (a linear function of $\mu$ ), and $L$ a linear operator in the surface coordinates. We have included a linear function for generality to allow energy sources and sinks, since the argument holds for this condition as well. The first two Jacobians in (3) and the Jacobian of $f$ and $\bar{\psi}$ vanish by virtue of the fact that $\bar{\psi}$ depends only on $\mu[\mathrm{Eq}$. (4) $]$ and, since we have chosen only one scale $n$, the Laplacian of $\psi^{\prime}$ is proportional to $\psi$, i.e., $\nabla^{\prime \prime} \psi^{\prime}=-n(n+1) \psi^{\prime}\left[\mathrm{EqL}_{\mathrm{S}}\right.$. (2) and (4)].

Let us first consider the time changes of the zonal components, $\psi_{m}(t)$. Nultiplying (3) by $P_{r}(\mu)$ and substituting the expansions from (4), we find, by utilizing the orthogonality properties of the surface harmonics, that all terms remaining on the right-hand side of (3) vanish on integration over longitude. This result is also a consequence of applying the selection rules for nonlinear interacting waves presented for the spectral vorticity equation by Baer and Platzman (1961). Noting, therefore, that the zonal field $\bar{\psi}(\mu)$ is invariant in time, let us consider the time changes of the wave components $\left(\psi^{\prime}\right)$.

We now multiply (3) by the conjugate of the surface harmonic for component $\gamma=n+i l_{\gamma}$, utilizing an asterisk to denote conjugation, and after introducing (4) integrate over the unit sphere. Expanding the Jacobians in tems of derivatives, periorming the differentiation with regard to $\lambda$ as indicated by (1), noting the time invariance of $\bar{\psi}$ and combining terms, we find that

$$
\frac{\partial}{\partial t} \psi_{\gamma}=\frac{i l_{\gamma} G_{\gamma}}{2 n(n+1)} \psi_{\gamma}
$$

where

$$
G_{\gamma}=\int_{-1}^{1} P_{\gamma^{2}}\left\{\left[\nabla^{2}+n(n+1)\right] \frac{\partial \bar{\psi}}{\partial \mu}+\frac{\partial f}{\partial \mu}+g(\gamma)\right\} d \mu,
$$

and $g(\gamma)$ depends on the linear operator $L$. The solution of (5) shows that each wave component $\gamma$ propagates at its own constant rate with frequency $\nu_{\gamma}=l_{\gamma} G_{\gamma} /$
$2 n(n+1)$, and the amplitude and consequently energy in each wave component is invariant with time. This conclusion is indeed the result stated earlier that wave components of given scale (n) will not interact nonlinearly with one another in a two-dimensional turbulent flow.

We shall show in Section 4, moreover, that growth of energy in spectral components due to barotropicbaroclinic instability tends to disappear simultaneously for all components of a given scale, when we utilize the index $n$ as the two-dimensional scale indicator.

\section{Data and representation}

To assess the utility of our proposed index and also to establish some spectral statistics of atmospheric data, we have selected to represent the wind field twice daily (0000 and 1200 GMT) for the months of January and February 1969 at five levels (7, 5, 3, 2.5, $2 \mathrm{db}$ ) in terms of their spectral components. The data were taken from both the NMC grid analyses and the tropical (Bedient) analyses, thereby providing complete hemispheric coverage. The two analyses were numerically merged by a linear weighting process and were then translated to the Kuri-grid (Kurihara and Holloway, 1967). ${ }^{2}$ The Kuri-grid has 4705 points on the hemisphere including the pole and equator; it is effectively a latitude-longitude grid, beginning with one point at the pole, incrementing $1.875^{\circ}$ in latitude, increasing the number of equidistantly spaced poirits on each latitude circle by four, and continuing to the equator with 192 points, thereby yielding the set of points on 49 equally spaced latitude circles.

Once the data are available on the Kuri-grid, they may be converted to a set of spectral coefficients; the method for performing this transformation has been detailed by Ellsaesser (1966a) and will only be outlined here. We have analyzed both the zonal $(u)$ and meridional $(v)$ wind components. In describing the generation of coefficients for the zonal wind, the reader should bear in mind that the meridional coefficients were derived in an identical manner. Given the data at longitudes $\lambda=j \Delta \lambda$ where $0 \leqslant j \leqslant p$ and $\Delta \lambda=2 \pi / p$, and at latitudes $\mu_{k}$ where $1 \leqslant k \leqslant 97$ to include the Southern $\mathbb{H}$ emisphere, we first expand about a latitude circle in Fourier series

$$
u\left(j \Delta \lambda, \mu_{k}\right)=\sum_{l} u_{l}\left(\mu_{k}\right) \exp (i l j \Delta \lambda),
$$

yielding by inversion

$$
u_{l}\left(\mu_{k}\right)=\frac{1}{p} \sum_{j} u\left(j \Delta \lambda, \mu_{k}\right) \exp (-i 2 \pi l j / p) .
$$

Note that we have suppressed time and height as independent variables although the functions do indeed depend upon them. The kinetic energy $E_{l}\left(\mu_{k}\right)$ at each

2 The merging and translation of this data were programmed and carried out for us by Dr. K. Miyakoda, GFDL/NOAA, Princeton, N. J. lititude the coeff

The tras of atmos $I_{i}$ is pre circles or latitude approact

The $g$ we have given as

$u(j$

where th the inver

In (11)

which 0 summati (see Flls

The to terms of

with the the $\alpha$-ter spheric ax from the

The equir generated fion of sa the total pansion ( wavenum distributi index is $\mathrm{p}$ expansion 
ergy

This

arave

wth

ipic-

usly

the

also

eric

icld

; of

2.5 ,

ata

the

ete

eri-

ere

llo-

the

ec-

ine

le,

its

he

of

ey

he

en

dd

d-

)n

35

dd

latitude circle $\mu_{k}$ in the l-wave maty be calculated from the coefficients generated by (8) and represented as

$$
E_{l}\left(\mu_{i}\right)-\frac{1}{2} u_{i} u_{l}^{*}+\frac{1}{2} w_{i}^{*} .
$$

The traditional approach to a spectral representation of atmospheric energy generally ends here. The energy $l i$ is presented as a function of $l$ for different latitude circles or a numerical integration of $E_{l}$ over a specified latitude belt is performed to yield averages, some approaching hemispheric.

The general expansion in solic harmonics of which we have so far completed only the longitudinal part, is wiven as

$$
u\left(j \Delta \lambda, \mu_{k}\right)=\sum_{\alpha} u_{\alpha} I_{\alpha}\left(j \Delta \lambda, \mu_{k}\right), \quad \alpha=n_{\alpha e}+i l_{\alpha},
$$

Where the functions $Y_{a}$ are delined by (1). Completing the inversion, we find

$$
\begin{aligned}
u_{\alpha} & =\frac{1}{2} \sum_{i} \omega_{k} u_{i}\left(\mu_{k}\right) P_{\alpha}\left(\mu_{k}\right) \\
& =\frac{1}{2 p} \sum_{j} \sum_{i} \omega_{k} u\left(j \Delta \lambda, \mu_{k}\right) Y_{k} *\left(j \Delta \lambda, \mu_{k}\right) .
\end{aligned}
$$

In (11) we hatve made use of the Numann weights which orthogonalyze the Legendre polynomials on summation; i.e., they are generated from the equations (see Ellsalesser, 1906)

$$
\sum_{k=1}^{q} \omega_{k} P_{\alpha}\left(\mu_{k}\right) P_{\beta}\left(\mu_{k}\right)=\int P_{\alpha} P_{\beta} \gamma=2 \delta_{\alpha, \beta} .
$$

The total kinetic energy over the surface is given in turms of the amplitudes of the coefficients $u_{a x}$ and $a_{\alpha x}$ as

$$
\begin{aligned}
E & =\int_{2}^{1} \frac{2\left(u^{2}+v^{2}\right) d A-\frac{1}{2} \sum_{\alpha}\left(u_{i x} u_{\alpha x}^{*}+v_{\alpha} i_{\alpha}^{*}\right)}{} \\
& =\sum_{\alpha} E_{\omega},
\end{aligned}
$$

With the energy in ach $\alpha$-component being given by the $\alpha$-term on the right-hand side of (12). The hemis)heric average in each planetary wave $(l)$ is determined from the total expansion by the sum over $n$, i.e.

$$
E(l)=\sum_{n_{i}} E_{\alpha} \approx \sum_{k} E_{i}\left(\mu_{i}\right)
$$

The equivalence in (13) of $l(l)$ with the sum of energies stenerated from (9) (which has been verilied by utilization of sample data) shows the correspondence between the total spectral expansion (10) and the partial exJunsion ( 7 ) when only the distribution with planetary wavenumber (a one-dimensional index) is desired. The distribution of energy in terms of a two-dimensional index is possible only by utilizing the complete spectral apinsion, which in terms of the index $n$ is griven by the sum

$$
E_{n}-\sum_{i \infty} E_{\alpha}
$$

It maly be noted that the spectral expansion requires data over the entire spherical surfice. Since we have data only over the Northern Hemisphere, some assumption on its distribution over the Southern Hemisphere must be made. Consistent with observation, we have assumed the zonal (iv) field to le symmetric and the meridional $(a)$ field to be antisymmetric across the equator. Calculations of the total energy and the energy in different scale groups for alternate choices of symmetry show negligible differexes from values arrived at by the above defined symmetry. The chosen symmetry does, however, eliminate any possible correlation in the rind components, effectively imposing a conclition of isotropy.

For analysis purposes, we have calculated separately the contributions from zonal and meridional energies, $E_{\alpha x}(u)$ and $E_{\alpha}(v)$, respectively, as well as the total rlefined by (12). Furthermore, we have calculated the energy in each planetary wave [see (13)] and in each two-dimensional scale component [see (14)]. Finally, these energies have been established at each available pressure level and for all time periods. For some analyses vertical averages of data sufficed. The averages were performed linearly with regard to pressure by first establishing values at 1 and 9 dt) through quadratic interpolation, assuming vanishing wind at zero and $1000 \mathrm{mb}$. These values, the $1-\mathrm{db}$ value depending on those at 2 and $2.5 \mathrm{db}$ and the 9-db value depending on the 7 - and 5 -db values, thereby served a double purpose, as they were needed for the linear analysis to be discussed in the following section.

Although our primary concern in this paper is with the energy distribution in terms of $l$ or $n$, the reader may be interested to see how the individual components $\left(E_{\alpha}\right)$ distribute. We have prepared for this purpose Fig. 3, in effect two spectral maps, with planetary wave index on the abscissa and zeros-in-latitude index on the ordinate, similar to the chart on the right-hand side of Fig. 1. On these maps we have plotted and analyzed the time-averaged and vertical mean kinetic cnergy as a percent of the total energy, for both the zonal and meridional winds. Of principal interest, one observes from these maps that for smaller scales (larger $n$ index) the isopleths of constant energy tend to follow lines of constant two-dimensional index considerably more closely than the coordinate lines which define the one-dimensional indices. This result may support the contention that the appropriate truncation for spcciral integrations should be triangular rather than rhomboidal (see Ellsaesser. 1966b), especially if one accepts the $n$ index as the appropriate $t w o$-dimensional index in the spherical domain. Nevertheless, a comparison of Fig. 3 with a similar figure describing a recent spectral integration of a general circulation molel (Bater and 

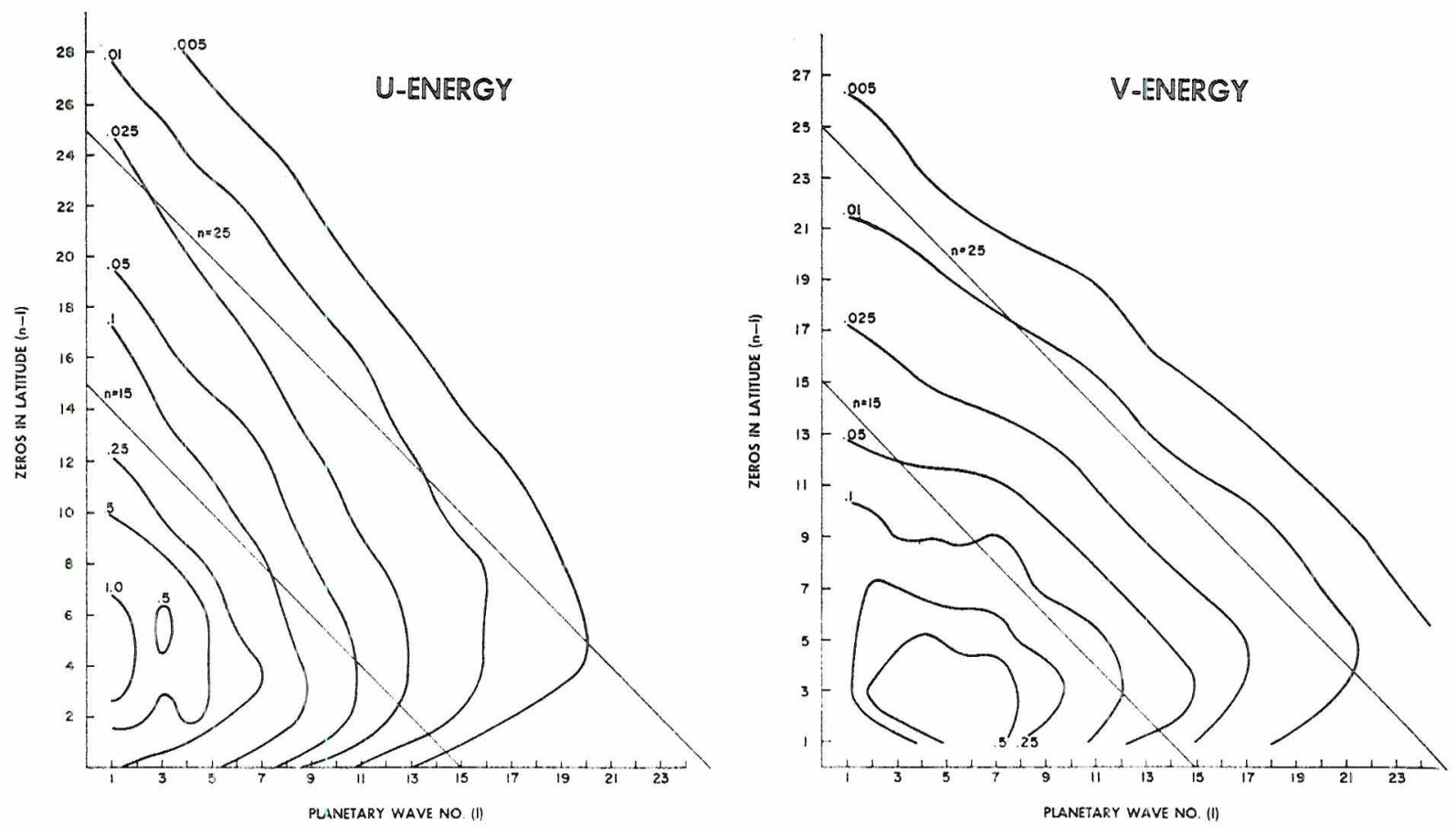

Fig. 3. Average kinetic energy (hoth in height and time) as a percent of total in each two-dimensional wave component, $\alpha=n+i l$, plotted and analyzed as a spectral map.

Alyea, 1971, Fig. 9) shows remarkable correspondence and suggests that with adequate spectral resolution even rhomboidal truncation will yield successful integrations.

\section{Linear analysis}

If we have selected a reasonable two-dimensional index, as argued in Section 2, we may hope to find some meaningful statistical distribution in our analyzed energy data as a function of that index. A correspondence of distribution in our data with the two-dimensional turbulence theory as predicted by Kraichnan (1967) would necessitate an investigation of the data distribution in the spectral region beyond the scale of forcing. Since we may relate the spectral domain of baroclinic instability with the region of forcing resulting from energy transformation, we must establish the baroclinically unstable wave region in terms of our two-dimensional index. If there exists a cutoff index value beyond which no instability exists, we might consider the spectral region beyond that point as exhibiting a two-dimensional turbulent structure predominantly driven by nonlinear momentum exchange, and with its energy source in the region of baroclinic instability.

In terms of the one-dimensional planetary wave index, most linear baroclinic stability studies (Charney, 1947; Eady, 1949; Phillips, 1954; etc.) and the more recent barotropic-baroclinic stability studies (Brown, 1969; Simons, 1970) indicate that the region of maximum instability is between waves $6-10$. Since current data accuracy deteriorates seriously for waves $l \geqslant 18$ [and even for longer waves according to Julian et al. (1970)], one is left with few waves to analyze for spectral statistics. Nevertheless both Julian et al. (1970) and Wiin-Nielsen (1967) find that their data show a logarithmic decrease of kinetic energy with planetary wavenumber with a slope near -3 .

To establish the corresponding longwave cutoff to instability (barotropic-baroclinic) in terms of our twodimensional index, we have solved the linearized potential vorticity equation in five atmospheric pressure levels $(1,3,5,7,9 \mathrm{db})$ with both the vertical and latitudinal zonal clistributions specified for each time period of available data as the zero-order state. This equation was solved in the spectral domain and each wave component was independently superposed on the ground state and its growth rate, if existent, was calculated. The model and its program were taken from Simons (1970) and will be outlined only briefly below; for details, the reader is referred to Simon's report.

The potential vorticity equation studied has the form (also discussed by Phillips, 1963)

$$
\begin{aligned}
& \frac{\partial}{\partial t}\left[\nabla^{2} \psi+f_{0}{ }^{2} \frac{\partial}{\partial p}\left(-\frac{1}{\sigma}\left(\frac{\partial \psi}{\partial p}\right)\right]\right. \\
& +J\left[\psi, \nabla^{2} \psi+f+f_{0}{ }^{2} \frac{\partial}{\partial p}\left(-\frac{1}{\sigma}-\frac{\partial \psi}{\partial p}\right)\right]=0,
\end{aligned}
$$

where the usual notation obtains; i.e., $\psi=\psi(\lambda, \mu, p, t)$ represents the stream field, $f_{0}$ is a mean value of the
Cori

bilit?

chosi

$(196$

horiz

defin

(15)

diffel

linea

zonal

wave

wher vects ject strea 

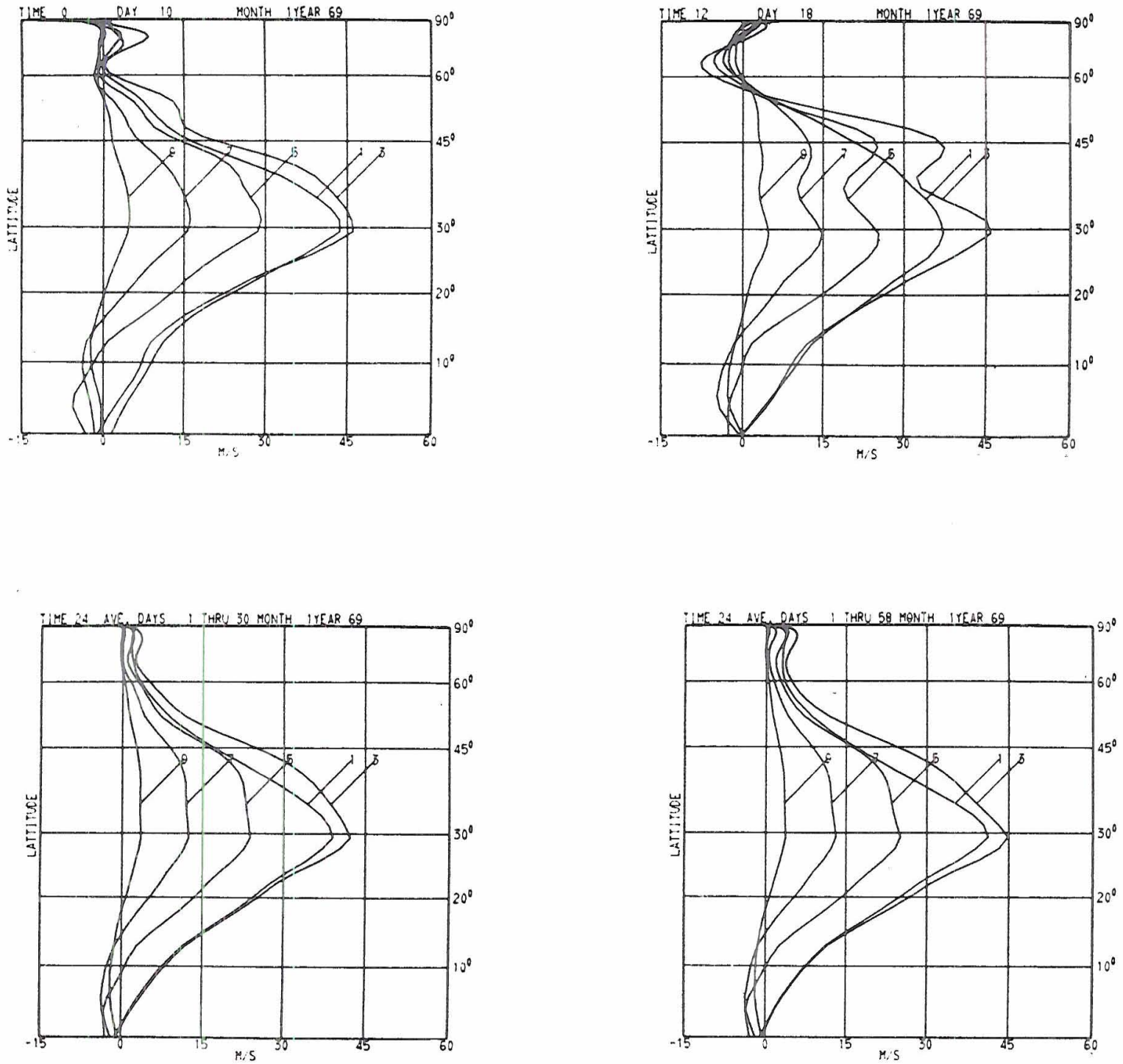

Frs. 4. Zonal wind profiles as a function of latitude and height (numbers on curves denote decibar levels) for two observation times and for two averaged periods.

Coriolis parameter, $\sigma=\sigma(p)$ is a standard static stability variable depending only on pressure with values chosen for average winter conditions given by Gates (1961), and pressure coordinates are utilized. The horizontal coordinates are lotitude and longitude, refined symbolically in Section 2, and as stated above, (15) is evaluated at five pressure levels with finite differencing used in place of pressure derivatives. The linearization hypothesis is imposed by considering the zonal field constant in time and introducing only one wave component; then, we have for the stream field,

$$
\psi=\psi_{0}(\mu, p)+2 \operatorname{Re}\left[\psi_{\alpha}(p, t) Y_{\alpha}(\lambda, \mu)\right],
$$

where $\psi_{0}$ is the zonal field and $\alpha$ represents any wave vector in the range, $1 \leqslant l_{\alpha} \leqslant 16$ and $l_{\alpha}<n_{\alpha} \leqslant l_{\alpha}+1.5$, subjuet to the constraint that $n_{\alpha x}+l_{\alpha x}$ be odd. The zonal stream field was determined by numerical integration of the stream equation

$$
\psi_{0}\left(\mu_{k}, p_{i}\right)=-\int_{0}^{\phi\left(\mu_{k}\right)} u_{0}\left(\mu, p_{i}\right) d \phi
$$

at all $k$ latitudes of the Kuri-grid and the five pressure levels considered-note here why it was necessary to interpolate values at 1 and $9 \mathrm{db}$ from the given data. The zonal expansion coefficients were then computed by the inversion method discussed in Section 3 from

$$
\bar{\psi}_{2 m-1}\left(p_{i}\right)=\sum_{k} \omega_{k} \psi_{0}\left(\mu_{k}, p_{i}\right) P_{2 m-1}\left(\mu_{k}\right)
$$

for $1 \leqslant m \leqslant 8$, thus yielding eight coefficients for each pressure level. The wave coefficients $\psi_{a}(p, t)$ may also be evaluated at each of the pressure surfaces, thus yielding a five-element vector when the values at the 

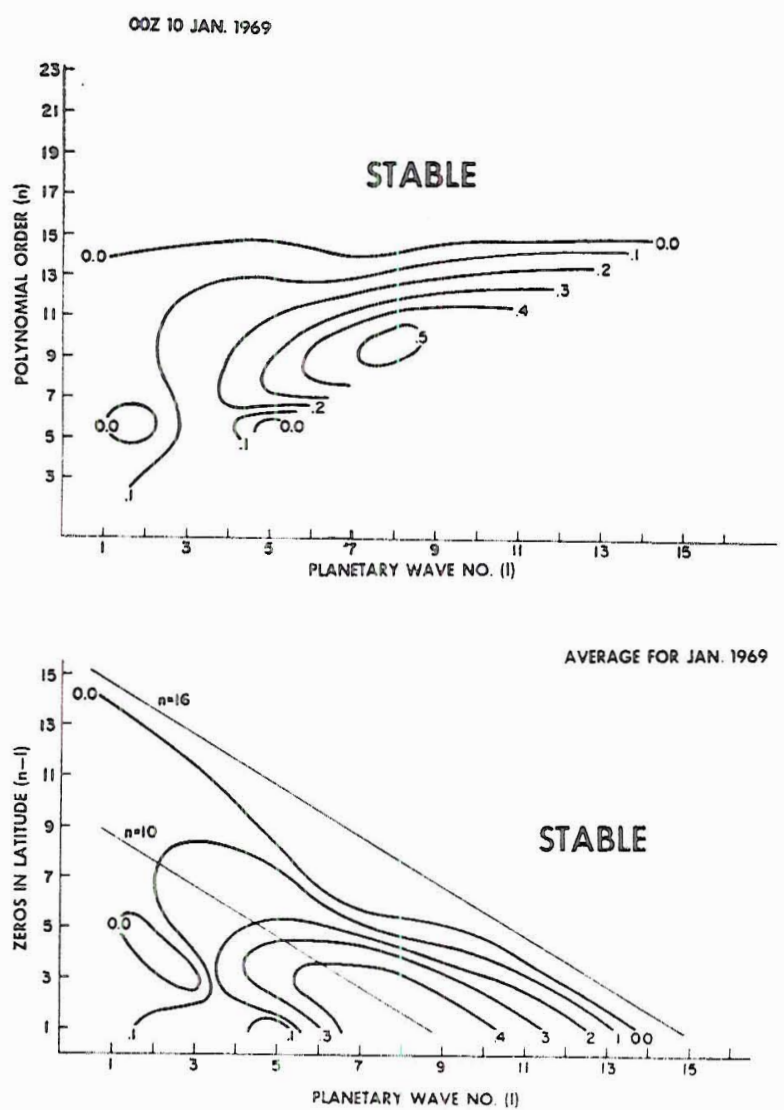
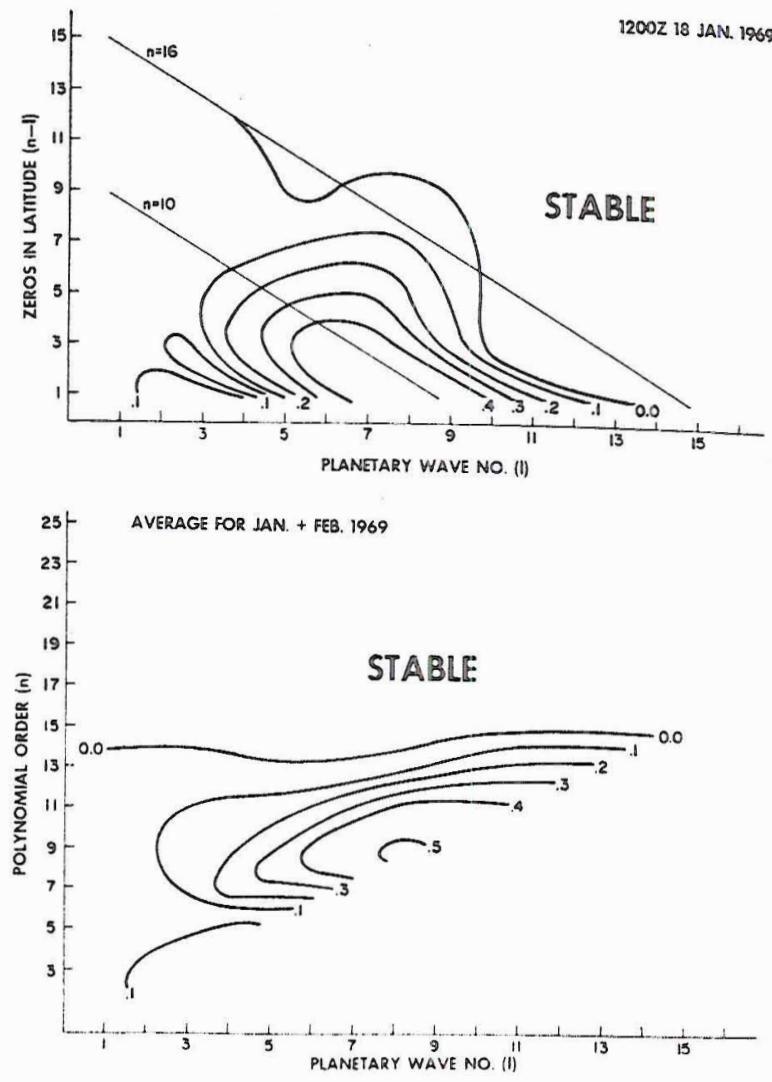

Fig. 5. Stability diagrams showing growth rates (e-folding time in inverse days) for wave components $\alpha$ interacting with the zonal distributions described in Fig. 4.

pressure surfaces are listed as

$$
\bar{\Downarrow}_{x}(t)=\left\{\psi_{\alpha}\left(p_{i}, t\right)\right\} .
$$

Substituting (19) and (18) into (15) in their appropriate series expansion, performing the necessary derivatives, and eliminating the horizontal space dependence by using the orthogonality properties of the Legendre polynomials (this process also involves the dis-allowance of self-interaction by the wave components), one arrives at the linear differential equation in time for the $\alpha$-wave stream coefficient,

$$
\dot{\bar{\psi}}_{\alpha}=i \mathrm{~A}_{\alpha} \overline{\mathrm{v}}_{\alpha} .
$$

The $(5 \times 5)$ matrix $\mathbb{A}_{\alpha x}$ depends both on the static stability distribution and the zonal stream coefficients $\bar{\psi}_{2 m-1}\left(p_{j}\right)$ for all allowed $m$ and $j$, which incorporate the vertical and horizontal structure of the zonal field. The roots of $\mathbb{A}_{\alpha}$ specify the stability of the $\alpha$ wave and its growth rate. The largest imaginary component is then plotted on an $(l, n-l)$ or $(l, n)$ diagram - both maps are used to familiarize the reader with these coordinates-and the calculation is performed for each wave component within the allowed range specified above; the plotted maps are then analyzed in terms of these growth rates. The process is repeated for each of the available data sets as well as for time averaged data.

Fig. 4 describes the zonal configuration for 0000 GMiT 10 January 1969, 1200 GMT 18 January 1969, averaged data for January 1969, and averaged data for January and February 1969, with the integers on the curves denoting decibar levels. We have chosen the two daily profiles because of their pronounced difference, one representing a single zonal jet, the other a double jet. The loss of the double jet at $100 \mathrm{mb}$ on 18 January is caused by the interpolation from the 250and $200-\mathrm{mb}$ levels. The corresponding growth rates associated with these distributions are shown in Fig. 5 , which represents the diagrams prepared from the stability analysis discussed above. The positions of the diagrams in Figs. 4 and 5 correspond in time; thus, one can readily assess the impact of zonal distribution on stability.

The regions marked stable show no wave growth and indicate that all waves for $n>16$ are effectively uninvolved in the baroclinic energy conversion process. The maximum growth rates (units of inverse $e$-folding time in days) appear for planetary waves $l=7-9$ in agreement with the one-dimensional theory. However, these growth rates apply principally to the lowest modes (largest scale) in latitude. Indeed, the decay of rowth isopleth: with is except f It appe: to baroc a reasor range $n$ growth substan the app alluded

5. Scal

We h Julian $\varepsilon$ spheric the vici in midd limit af the resi to thre would $\mathrm{k}$ the qua we do 1 two-din are ider

Let r planeta the rest of inder is comI shorter in latit index $n$ is that shorter been aj the poi

The tribute may be Fig. 1. waves scale $n$ which : This n1 total a ponent: no cor: require acceptz tabulat (also c shows 1 accepte ponent: 
whoth rate with index is of stch a nature as to yield Wripleths of zero growth which correspond very closely. wh isopleths of constant two-dimensional scale $n$ a...pt for some deviation in the case of a double jet. It appears, therefore, that a cutoff index value related 1. buroclinic processes does exist in our data, whd that a cisonable cutotf index vilue may be chosen in the range n, $=14-16$. This result, the confuence of zero snwth with a constant value of index $n$, tends to sthatintiate our argument for selecting this index as lic appropriate two dimension index, an arment intarted 10 in Section ?

\section{Scale limit on data accuracy}

We have already indicated, based on the analysis of Julian of al., that the reliability limit of current atmowheric datia in terms of planetary wavenumber is in the vicinity of $l=10-18$, corresponding to wavelengths in middle latitudes of $1500 \mathrm{~km}$. Should this numerical limit apply also to the two-dimensional index $n$, using the results of the previous section for a lower cutoff to thret-dimensional forcing, virtually no scale data would be avalable to test for statistical equilibrium in the quasi two dimensional spectral range. Fortunately, wi do not believe that the limit of resolution for the 1wo-dimensional index and the planetary-scale index are identical, as we shall now attempt to show.

Let us set the limit to reliability of data in terms of plimetary scale at wavenumber 18 , based essentially on the results of Julian $\mathrm{a}$ al., but with a slight relaxation of index value. On the assumption that data reliability jis comparable in both latitude and longitude for the shorter scales, we shall also set the limit of reliability in latitude, represented by the one-dimensional scale index $n-l$, at 18 . The implication of these scale limits is that, for both latiundinal and longitudinal waves shorter than wavenumber 18 , serious smoothing has been applied which conseguently distorts the data to the point where interpretation becomes questionable.

The number of wave components $\alpha$ which contribute to a single two-dimensional scale $n$ is $n+1$, as may be clearly seen from an $(1, n-l)$ diagram such as Tir. 1. Indeed, Fig. 2 gives an example of the included Haves for $n=5$, also describing their nodes. For any sile $n$, one maly calculate the number of components which are in the range of allowed scilles, $l, n-l \leqslant 18$. This number is $37-n$ and must be compilied to the wal number $n+1$. Clearly for scales $n \leqslant 18$ all comIronents are in the acceptable range, whereas if $n \geqslant 37$, no components have acceptable data according to the aruirements set forth above. The ratio (percent) of areptable components to total components has been tabulated in Table 1 for two-dimensional scale values also denoted as ordinal index) of $18 \leqslant n \leqslant 29$, and hows that for $n-29$ only $27 \%$ of the components are areptable. Howcver, nearly half or more of the comfonents may be acceptable for values of $n \leqslant 25$. In
TALLE 1. Percent of waves with acceptable olservational data in each of the ordinal index groups for $18 \leqslant n \leqslant 2$ ) together with the percent of acceptalile encrgy at three pressure levels.

\begin{tabular}{|c|c|c|c|c|}
\hline $\begin{array}{l}\text { Ordinal } \\
\text { index }\end{array}$ & $\begin{array}{c}\text { Percent } \\
\text { of wave } \\
\text { with } \\
\text { m is }\end{array}$ & \multicolumn{3}{|c|}{ Petcent nerpy in ware with $m \leqslant 18$} \\
\hline 18 & 100 & 100 & 100. & 100. \\
\hline 1) & 90 & $97.2 \pm 3.2$ & $96,0 \pm .1 .9$ & $8(0.7 \pm 9.2$ \\
\hline 20 & 81 & $89.3:=7.0$ & $88.0+9.3$ & $88.2 \pm 0.8$ \\
\hline 21 & 72.7 & $81.8=8.5$ & $770 \div 9.1$ & $66.6 \pm 9.3$ \\
\hline 22 & 65.2 & $77.3 \pm 9.9$ & $77.4 \div 9.4$ & $71.7+10.2$ \\
\hline 23 & 58.3 & $60.5 \pm 9.7$ & $70.5 \pm 9.7$ & $05.7+11.2$ \\
\hline 2.4 & 52. & $57.8 \leq 10.9$ & $58.8 \div 11.8$ & $5.4 .9+10.2$ \\
\hline 25 & 46.2 & $5.3 .2 \pm 11.3$ & $54.3 \pm 11.1$ & $43.6 \pm 10.2$ \\
\hline 26 & $\$ 0.7$ & $40.0 \pm 10.8$ & $47.5+11.6$ & $42.0 \pm 11.1$ \\
\hline 27 & 3.5 .7 & $40.1 \pm 0.4$ & $41) .1 \pm 8.8$ & $30.4 \div 8.0$ \\
\hline 28 & 31 & $35.9 \pm 8.7$ & $38.0+11.0$ & $30.9 \pm 9.2$ \\
\hline 29 & 20.7 & $33.7+10.6$ & $32.6 \pm 9.5$ & $28.1+0.3$ \\
\hline
\end{tabular}

Table 1 , $m$ represents both $l$ and $n-l$, i.e., both must satisfy the conditions $m \leqslant 18$.

Since data are available for a two-month period, we may also determine the percent of clata in the acceptable scale range, a value which will undoubtedly differ from the percent of acceptable components listed in Table 1. After time-averaging the kinetic energy in each wave component for all data at levels 700,500 and $200 \mathrm{mb}$, we compute and list in Table 1 the percent of energy data in the acceptable scale range $(m \leqslant 18)$ to the total energy, together with the standard deviation for the same range of scale values, $18 \leqslant n \leqslant 29$. We note from the table that in excess of $50 \%$ of the data is acceptable for scales $n \leqslant 25$ except at $200 \mathrm{mb}$.

Based on the results of this section and the last, we propose that a reisonable scale range for testing twodimerisional turbulent encrgy exchange from current atmospheric data is $14 \leqslant n \leqslant 25$. In this region we may anticipate both no baroclinic energy transformation and rasonably accurate observational data. Analyses of data to be presented subsequently will substantiate this contention.

\section{Spectral distribution}

To describe the energy in cifferent scale components as a function of the scale index, we have prepared several charts (Fig. 6) wherein eneroy amplitude is plotted against wavenumber on a log-log scale. The energy groups include both $E(l)$ and $E_{n}$ for comparison [see Eqs. (13) and (14) for definitions] with $E$, plotted above $E(l)$ on each chart. We have computed the zonal and meridional energies separately, defined as $U$ and $V$ respectively, and presented them as well as the total energy, $L+V$. Three levels, 7,5 and $3 \mathrm{db}$, are represented and the heavy curve characterizes the vertical mean. The periods chosen correspond to those utilized in the linear analysis presented in Section 4 . Solid lines denote the -3 slope.

A casual glance at Fig. 6 will show that we have plotted energy in the scale range $5 \leqslant l, n \leqslant 40$. However, our previous assessment of dala accuracy dearly im- 

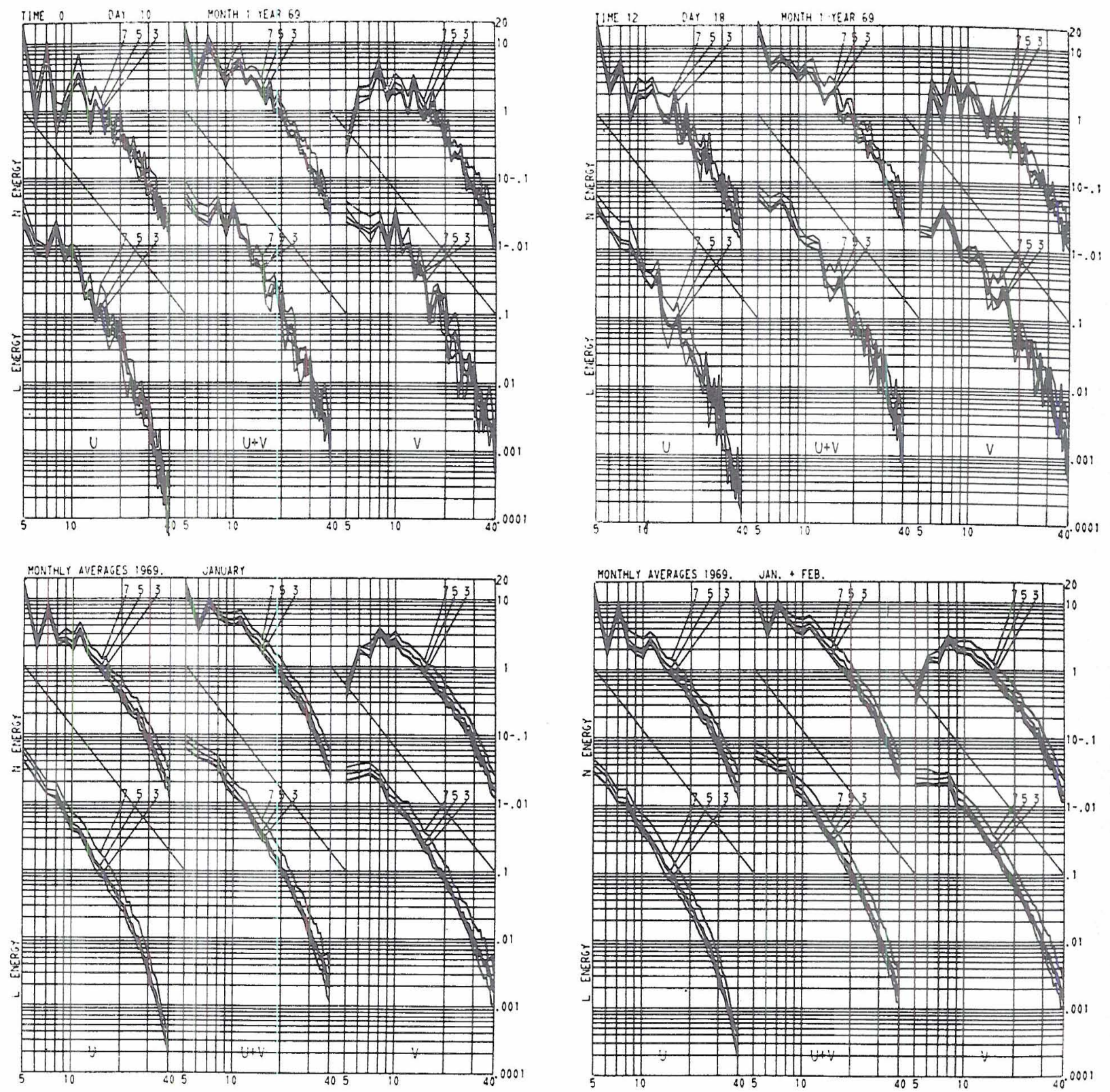

FIG. 6. Spectral distributions of zonal $(U)$, meridional $(V)$ and total $(U+V)$ kinetic energy for the vertical mean and decibar levels $3,5,7$, for the two-dimensional index $(n)$--upper curves-and the planetary wave index $(l)$--lower curves. Sections a-d correspond to dates utilized in Figs. 4 and 5 . Solid lines denote the -3 slope.

plies that much of the data presented, indeed that for $l>18$ and $n>25$, is not representative. Since these extra data are available from our source, namely NMC, and are utilized as initial data in the NMC numerical prediction model, we attempt to show in Fig. 6 not only atmospherically relevant energy distributions, but also those distributions consequent on smoothing which are utilized in numerical forecasting. Lest the reader misinterpret this presentation as a criticism, this writer feels that little is known about the influence of the shortest scales in a numerical prediction model, especially as a function of the time period of integration, and the availability of actual initial conditions utilized may assist in assessing the impact which the shortest scales have on predictability in terms of their spectral distribution. Our predominant interest in the shorter, non-baroclinically influenced, scales led us to neglect the longest waves, those less than wavenumber 5. All amplitudes in this and subsequent analyses are normalized by the total energy and the results are presented in percent.

On comparing Figs. $6 \mathrm{a}$ and $6 \mathrm{~b}$ with $6 \mathrm{c}$ and $6 \mathrm{~d}$, one is immediately impressed with the variability in energy from one wavenumber to another, a variability which effectively disappears on time averaging. Although these fluctuations are large, the energy amplitude does

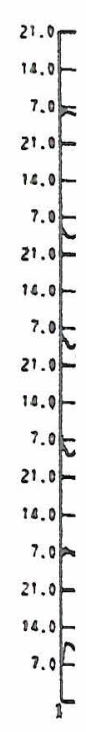

Fig. scales dimens heights Februa for fur:

tend : decay quent range the $d$ sentin at lec slope ble, a: applie this in accur:

We temat ceeds corrol per le cyclor Since 


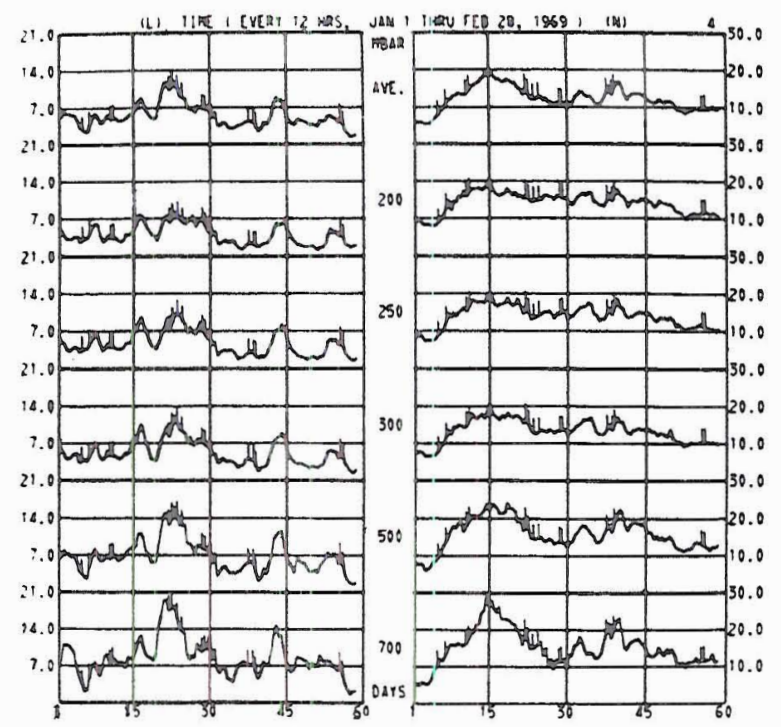

Fic. 7. Time dependence of wave energy as a percent of total in ales with indices $4,7,15$ (7:ac, respectively), for both onedimensional $(l)$ and two-dimensional $(n)$ indices for all observed eeights and the vertical mean. Time runs from 1 January to 28 Fobruary, 1969, with pip marks denoting missing data. See text inr further discussion.

and to decay with watenumber, and statistics of this Jecaly will be presented in quantiative form subseiuently. Moreover, there is some indication, in the ange of data acceptability as previously defined, that the distribution of energy approaches the line representing -3 , if not for the plantary wave distribution, at. least for that of our two-dimensional index. The lope steepens sharply for scales shorter than acceptable, an indication that perhaps the smoothing operator upplied to the data tends to remove energy; however, ihis interpretation must be subjected to test with data iccurate in the shortest scales.

We note further that the energy amplitude is syscmatically larger for all described waves as one proeeds to pressure levels closer to the ground. This result orroborates our more qualitative observation that upper levels appear more zonal than lower levels and that yclone amplitudes are relatively larger at the surface.
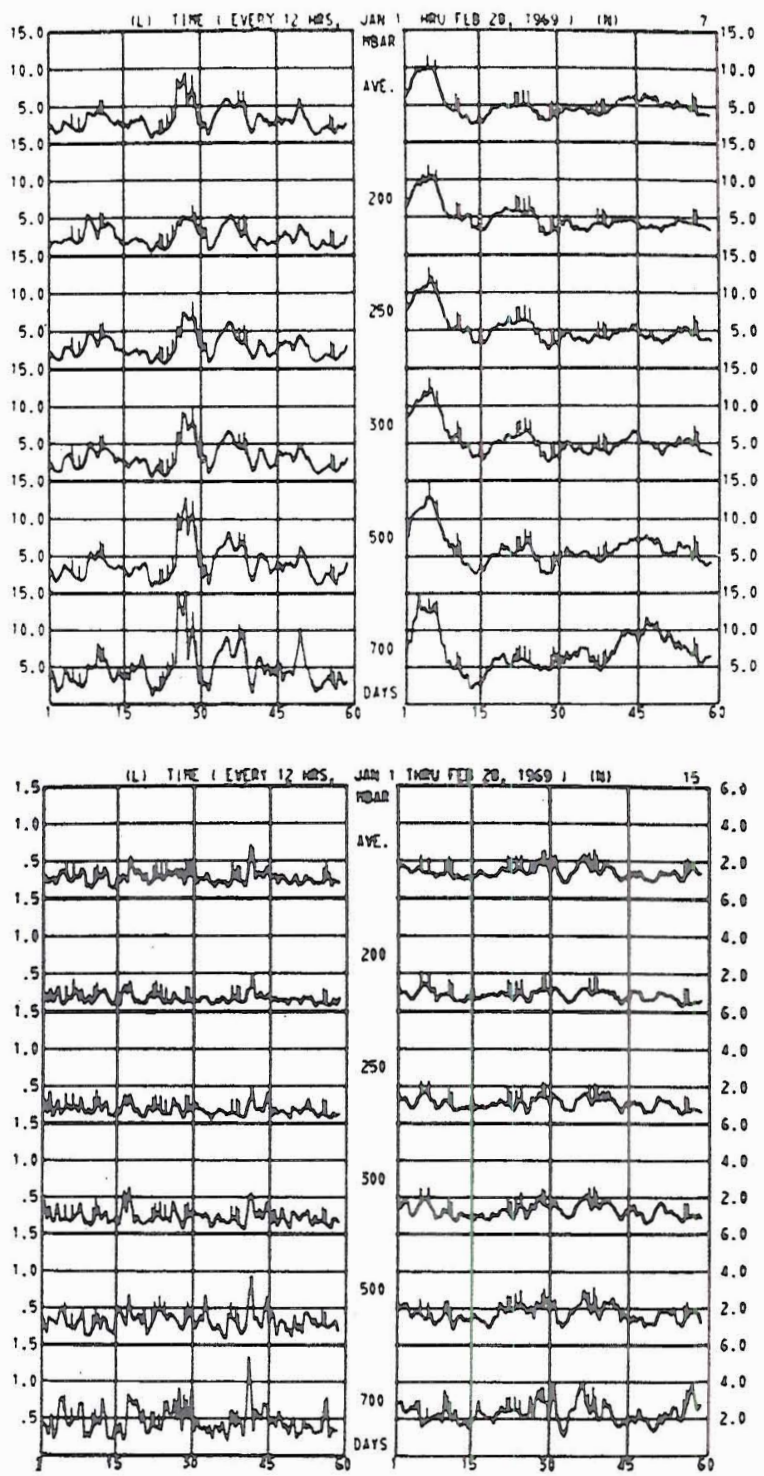
Since our data are nomalized there is no indication here of absolute amplitude; indeed the higher levels do attain larger energy amplitudes, of which the longest waves of planetary scale have a disproportionate share.

Perhaps the most interesting observation from Fig. 6 is the significantly larger energy amplitude for all but the largest scales in the $n$ relative to $l$ index, implying that wave for wave, more energy resides in the twodimensional scale. Although we do not propose to explain this fact, we do note that each two-dimensional scale includes a number of planetary scales [see Eq. (14)]. This effect holds true for the $U$ and $V$ components of the energy as well as the total energy although, whereas the $U$ and $V$ components show an equipartition of energy in the two-dimensional scales outside of the actively baroclinic region, the $V$ components show larger amplitudes than the $U$ components in the planetary wave distributions. The latter observation may add another positive argument for selecting $n$ as the appropriate scale index. 

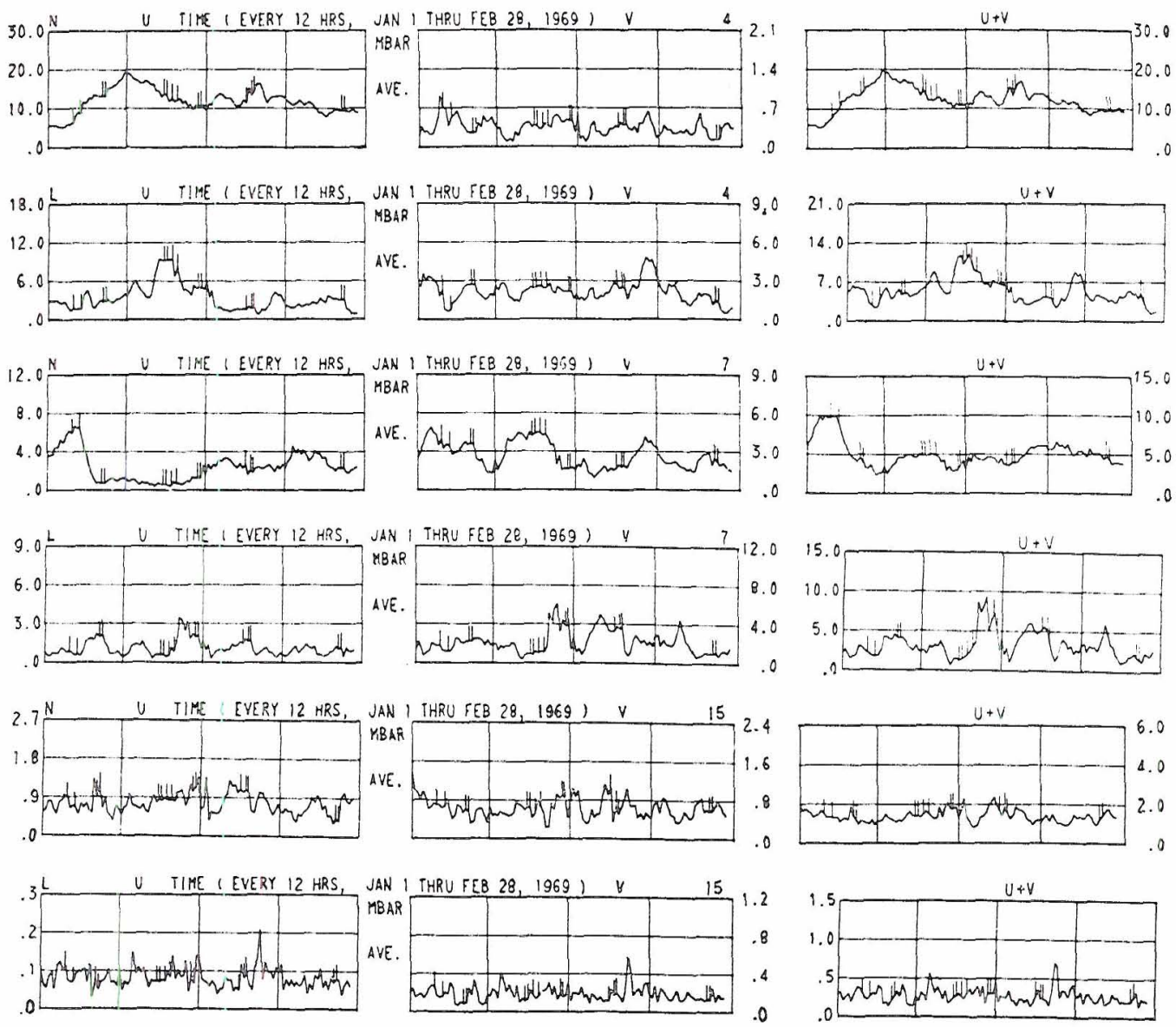

FIG. 8. Time dependence of vertical mean energy as a percent of total for waves $4,7,15$, for both $l$ and $n$ describing the contributions from the zonal $(U)$ and meridional $(V)$ components.

\section{Time variation of energy by scale}

Let us now turn our attention to the detailed variation with time of the energy in the individual scale groups, $E(l)$ and $E_{n}$, their distribution with pressure, and their $U$ and $V$ component contributions. Since it is impossible to show all scales, we have selected three waves to represent what might be considered long, medium and short waves and given, respectively, by index numbers 4, 7 and 15. On Fig. 7 we present the time variation of these three waves--Fig. 7a for wavenumber $4,7 \mathrm{~b}$ for 7 , and $7 \mathrm{c}$ for 15 --the left-hand chart representing the planetary index $(l)$ and the right side depicting the ordinal index $(n)$. The abscissa denotes time in days extending over the period from 1 January to 28 February 1969, and the ordinate represents component energy as percent of total for each of the five observed levels and the vertical mean (the uppermost curve). Pip marks on curves of this and subsequent figures indicate times when clata were unavailable.

We see from this figure that the oscillations for the $l$ and $n$ indices are uncorrelated in time, regardless of scale. Although such a result is perhaps obvious for the longer waves, on the assumption of isotropy in the wave domain for the smaller scales, one might anticipate some correlation between the one and twodimensional representations. That the observations do not bear this correlation out indicates that for the indices chosen, the one-dimensional index (herein the planetary wavenumber) is not a suitable substitute for describing the two-dimensional scale characteristics. This fact will also be apparent from the remaining presentation. As noted also on Fig. 6, the amplitude of the energy in the ordinal index is significantly larger than that in the planetary incex for the shorter scales, an observation which is true at all atmospheric levels.

Whereas we have seen from Fig. 6 that the relative energy amplitude in the waves is larger at higher pressure levels (closer to the surface), we see from Fig. 7 that this result occurs in conjunction with larger amplitude fluctuations near the surface, although there is no significant change in the frequency of oscillation. One may conclude that energy exchange processes are more pronounced at lower levels, an effect undoubtedly related to the proximity of the viscous boundary layer. Although the energy fluctuations at higher levels are less pronounced than near the surface, they are by no means negligible; indeed, perhaps the principal ob- serv

of 1

erra

the

seen

the

only

indi.

thos

with

rela:

com

the

nent

wav

of el

inde

the

tude

scalt

ener

apps

$T_{1}$

pres

of $t$ ]

incli

L, I

cally

ordis

valu.

7 an

the

shor

$V$ c

amp.

than

the 5

Fig. sente 
TASLI 2. Time averiges and standard deviations of percent energy in scale components $0 \leqslant l, n \leqslant 15$ with contributions from the zonal $(C)$ and meridional $(V)$ parts.

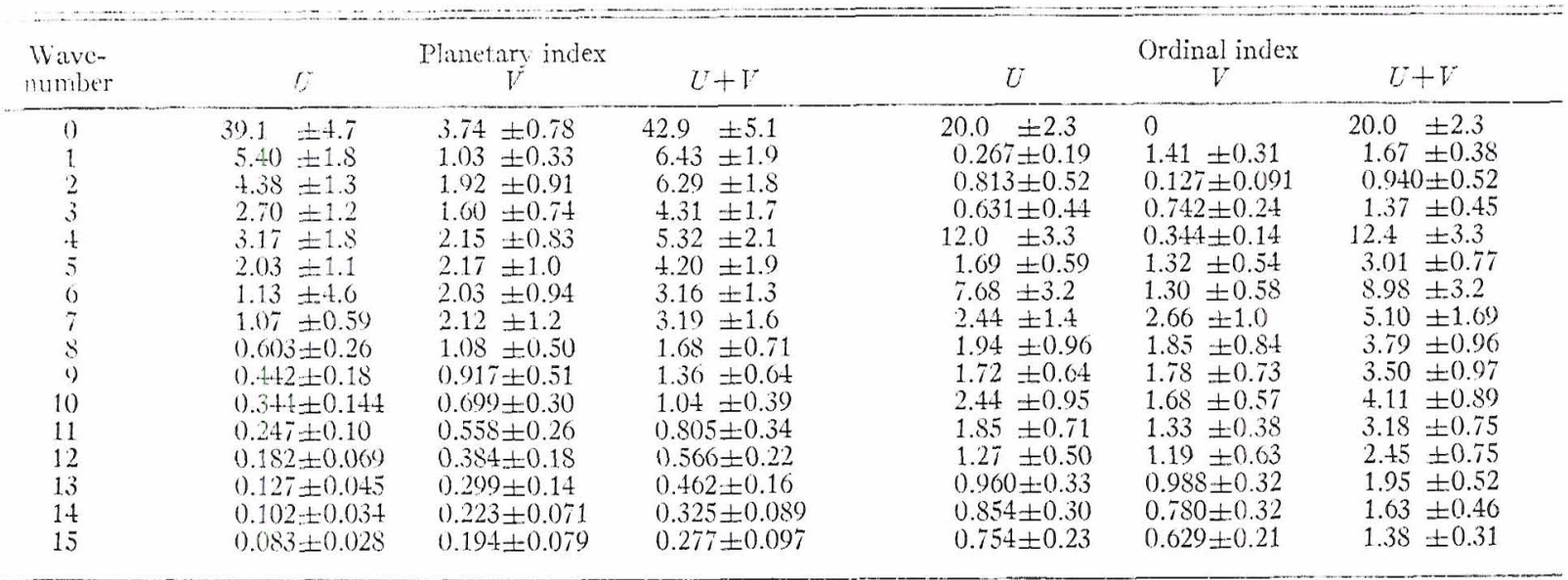

srration to be made from. Fig. $T$ is the non-constancy if the scale energy in time and its significant and aratic variability.

The relative infuence of the $L$ and $V$ components to the time variation of the total scale energy may be xen from Fig. 8. Because we have already observed the variation in pressure from Fig. $\tau$, we present here mly the vertical mean distributions for scales with indices 4,7 and 15 and with coordinates identical to those on Fig. 7, i.e., the abscissa denotes time in days with 15 days per interval. As before, we note no cordation between the $l$ and $n$ elements of the wind omponents. For the long wave, with inder equal to 4 , the energy is predominantly in the zonal $(U)$ component, especially for the two-dimensional index. The waves with indices 7 and 15 show roughly equipartition defining the deviation limits of the time averaging. Although the variability is again seen to be large, there is a tendency toward a -3 slope in part of the scale region, a tendency which will now be cxplored in greater detail.

\section{Energy distribution by scale}

Our observations of time mean energy distributions (Firs. 6 and 9) suggest that a power relationship may exist between the energy data and the scale index in the form

$$
E_{m}=A m^{b}
$$

where $m$ represents the scale index ( $l$ for one dimensional and $n$ for two dimensional), $A$ and $b$ are constants, and $E_{m}$ represents the energy in the $m$-scale component. In (21), b clearly represents the slope on a $\log -\log$ graph of $E_{m}$ with $m$, and describes the ob-

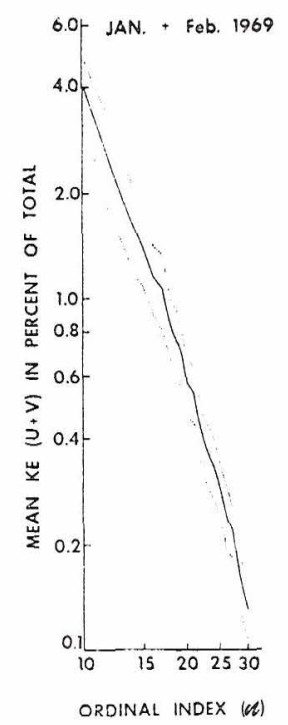

Frs. 9 Time and vertical mean kinetic energy with standard deviation in two-dimensional scales $10 \leqslant n \leqslant 30$. 


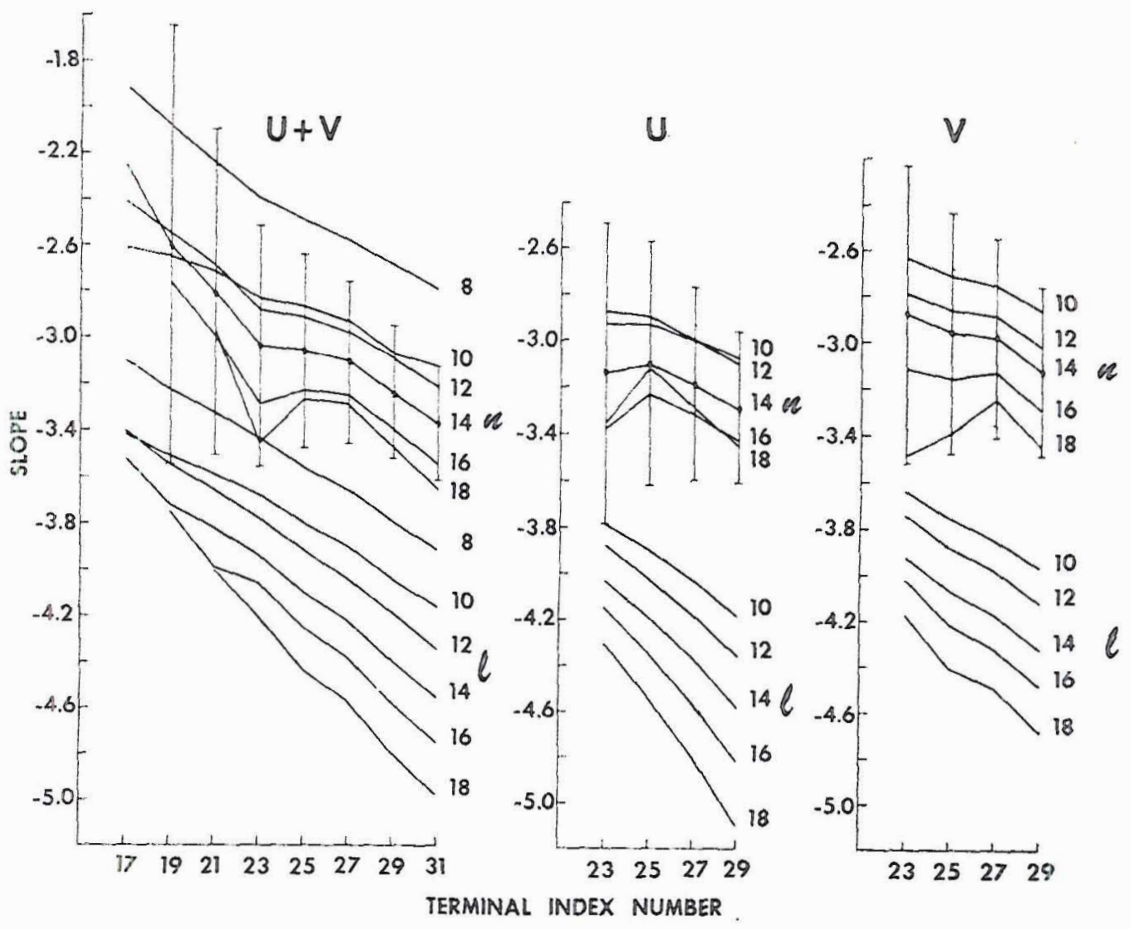

Fig. 10. Statistical slope of energy with scale (both $l$ and $n$ ) for different spectral regions. Alsscissa denotes terminal index value and number following curve denotes initial index. Slopes of meridional and zonal components are also represented.

servational analog to the energy-scale relationship anticipated from two-dimensional turbulence theory. If we define the scale region over which $b$ is to be established from data by $m_{i} \leqslant m \leqslant m_{l}$, where $m_{i}$ is the initial scale index and $m_{t}$ the terminal index, and all $N=m_{t}-m_{i}+1$ values in the allowed scale range are utilized, then by the process of least squares the data may be fit to the function (21) yielding $b$ in terms of $m_{i}$ and $m_{l}$, i.e.,

$$
b=\frac{N \sum\left(\ln m \ln E_{m}\right)-\sum \ln m \sum \ln E_{m}}{V \sum(\ln m)^{2}-\left(\sum \ln m\right)^{2}} .
$$

The sunmations in (22) go over the allowed range of $m$ as defined above.

The scale limits within which we might discover distributions (slopes) comparable to those anticipated from turbulence theory have been defined in Section 5 . Nevertheless, both because we have data over a wide scale range (and utilized as initial conditions for numerical integration of atmospheric models), we have chosen to calculate $b$ for $a$ wide range of values of $m_{i}$ and $m_{l}$. We show in Fig. 10 some values of slopes $(b)$ for vertical mean time-averaged data, for the $U$ and $V$ energy components as well as the complete energy, and for both indices $l$ and $i l$. The abscissa represents the terminal index $\left(m_{t}\right)$ and the numbers to the right of each curve specify the initial indices $\left(m_{i}\right)$.

It is immediately apparent, in terms of meaningful atmospheric data, that only a few points on all the curves describing the $l$-scales are applicable, those points having values $l_{i}=8,10,12$ and $l_{t}=17$. In terms of previous investigations with this procedure, the point $l_{i}=8, l_{t}=17$ approaches a value $b=-3$ and compares favorably with the calculations of WiinNielsen (1967) and Julian et al. (1970). The remaining values for the l-scales indicate the effect of smoothing on the initial data, and highlight the rapid reduction with scale of the zonal $(U)$ energy relative to the meridional $(V)$ energy, an observation noted previously.

Diverting our attention now to the slopes computed for the two-dimensional scale index $(n)$, we note that for the acceptable index region previously defined, $14 \leqslant n \leqslant 25$, the slope values are close to -3 , and indeed do not vary significantly for variations of the terminal index in the range $23 \leqslant n_{t} \leqslant 27$. If one proceeds into the actively baroclinic region, $n_{i}<14$, the slope becomes less steep; if one neglects some baroclinically inactive two-dimensional scales, $n_{i}>14$, the slope becomes more steep. These results thus tend to corroborate our earlier contention that the range $14 \leqslant n \leqslant 25$ is the most reasonable for testing with respect to two-dimensional turbulence theory. A systematic difference between the slope of the $U$ and $V$ contributions does appear, with the zonal $(U)$ component energy decreasing somewhat more rapidly with scale.

Although the slope values described herein are promising by comparison to turbulence theory, large variability in time exists for these values (already antir

\section{fiden}

$n_{i}=$

great

of $\mathrm{sl}$

the

inclu

to er

a va.

level

some

trary

there

betw

larges

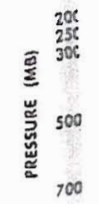



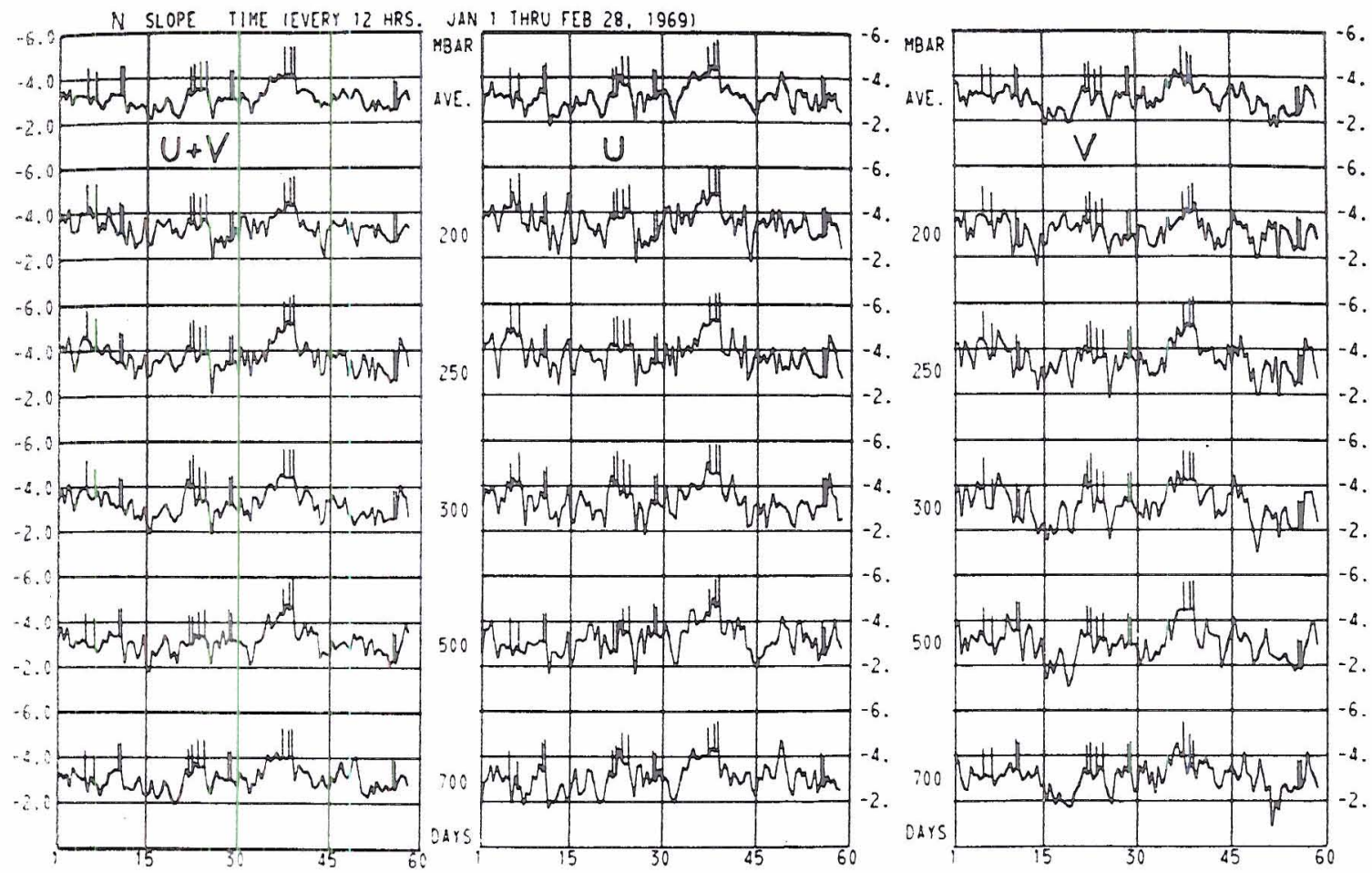

14. 11. Time variation of slopes in the scale region $14 \leqslant n \leqslant 25$ for all reported levels and vertical mean, with contributions from the $U, V$ components. Time period is the same as in Figs. 7 and 8 .

hose erms

the

and

Viin-

ning

hing

tion

the

isly.

ited

that

red,

and

the

ro-

the

iro-

the

to

lge

ith

ys-

V

)o-

ith

ire

ge

1y. anticipated from Fig. 0) and is expressed by the confidence limits (deviations) drawn for all points on the $n_{1}=14$ curve of Fig. 10. This variability is described in weater detail in Fig. 11 which shows the time variation of slope (b) for the range $14 \leqslant n \leqslant 25$, including both the $i$ and $V$ contributions, and all reported levels including the vertical average. It is hardly necessary to cmphasize the large amplitude variability in time, a variability which cloes not differ from one pressure level to another, and which may actually amplify somewhat for the individual $U, V$ contributions. Contrary to our observation of the energy fuctuations, there appears to be some positive correlation in slope between the $U$ and $V$ components, principaliy for the larger time period fluctuations which, if significant

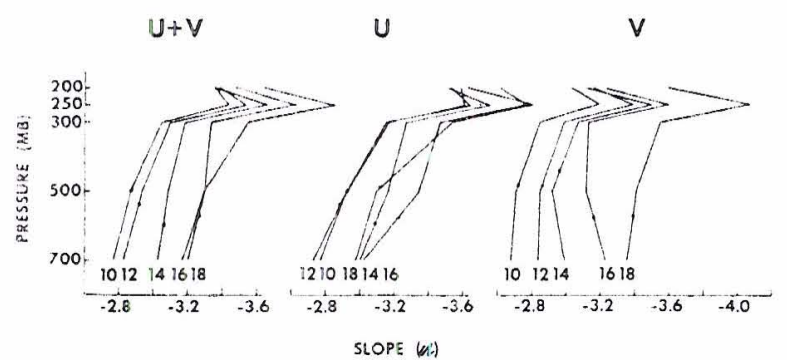

Fici. 12. Vertical variation of statistical slope (time averaged) for the two-dimensional index with terminal value of 25 and initial value at hottom of curve. Both energy components $(U$ and i) are described and the dot on each curve denotes the vertical itverage. enough, might allow the use of either of the contributions as an indicator of the total fuctuation.

Fig. 11 also suggests a steepening of slope with height in the atmosphere. This effect is shown more clearly in Fig. 12 where we have plotted the slope for the $n$-index in the range of $n_{i}$ specified at the bottom of the curves and terminating at $n_{1}=25$ (similar results were found for $n_{t}=23$ and 27 ). The dot on the curves denotes the slope of the vertical mean data, all data utilized here having been averaged in time. One sees again, as in Fig. 10, the lower values of slope for the $V$ relative to the $U$ component of energy. The result of primary interest seen, however, is the significant increase in slope with altitude, especially at the $250-\mathrm{mb}$ level. One is again led to the interpretation of weaker wave activity at higher levels, but the predominance of the 25()$-\mathrm{mb}$ level with regard to its steep slope is as yet unexplained.

\section{Conclusion}

To draw an analogy between guasi two-dimensional atmospheric flow in a spherical surface with the theory of two-dimensional turbulence, we have presented a scale representation based on the expansion of atmospheric variables in spherical harmonics. The twodimensional scale index in this case is given by the order of the Legendre polynomials, and we have shown that this index is not only appropriate in terms of dimensional analysis, but that walves of the satme scale 
will not interact nonlinearly with one another in a turbulent flow.

We have analyzed two months of wind data at five pressure levels and described the resulting kinetic energy in terms of the proposed inder. Baroclinic stability calculations show that beyond inder values of 14 , little instability occurs and the remaining spectrum may be considered quasi two-dimensional. Further analysis of the data in terms of accuracy suggests that meaningful calculations may be performed with current data to index values of 25 . Considering the energy distribution of our data sample as a function of the index under consideration, and in the acceptable region of quasi two-dimensionality, we find a logarithmic decrease of energy with a slope near - 3 , in correspondence with the expectations from the theory of two-dimensional turbulence.

Based on this result we recommend that the proposed two-dimensional representation be adopted for describing atmospheric fields and be considered in the development of suitable parameterization of the shorter scales. A word of caution, however, is in order with this latter recommendation. Although the statistics on data distribution correspond well with theory, the data also show large variability in time. Whether this time variability is of importance in affecting the larger scales or may be eliminated by parameterization is rot clear and must be explored.

Acknowledgments. This research was begun while the writer was a visitor at the Geophysical Fluid Dynamics Laboratory, Princeton, N. T., and much of the data analysis was completed after that visit through the assistance of Dr. E. M. Rasmusson. During that period substantial computer time and aid was provided by GFDL/NOAA. The merging of the NMC and tropical analyses and the subsequent conversion to the Kuri-grid were prepared and carried out under the direction of Dr. K. Miyokoda. The linear model utilized in the stability calculation was cleveloped and programmed by Dr. T. J. Simons. A portion of this research was supported by the Atmospheric Sciences Section of the National Science Foundation under Grant No. GA-11637 to Colorado State University.

\section{REFERENCES}

Baer, F., and F.N. Alyea, 1971 : Effects of spectral truncation on general circulation and long-range prediction. J. Atmos. Sci., $28,457-480$.
-- and G. W. Plat:man, 1961: A procedure for mumerical integration of the spectral vorticity equation. $J$. Metcor. $18,393-401$

Brown, J. A., Jr., 1969: A numerical investigation of hydrodynamic instability and energy conversions in the quasigeostrophic atmosphere. $J$. Atmos. Sci, 26, 352-375.

Charney, J. G., 1947: The dynamics of long waves in a baroclinic westerly current. J. Meterr., 4, 135-162.

Eady, E. T., 1949: Long waves and cyclone waves. Tellus, 1 , $33-53$

Ellsaesser, H. W., 1966a: Expansion of hemispheric meteorological data in antisymmetric surface spherical harmonic (Laplace) series. J. Appl. Meteor., 5, 263-2خ6.

$\ldots$ - 1966b: Evaluation of spectral versus grid methods of hemispheric numerical weather prediction. J. Appl. Meterr., 5, 246-262.

Gates, W. L., 1961: Static stability measures in the atmosphere. J. Meteor., 18, 526-533.

Julian, P. R., W. M. Washington, L. Hembree and C. Ridley, 1970: On the spectral distribution of large-scale atmospheric kinetic energy. J. Atmos. Sci., 27, 376-387.

Kraichnan, R. H., 1967: Inertial ranges in two-dimensional turbulence. Plys. Fluids, 10, 1417-1423.

Kurihara, Y., and J. L. Holloway, Jr., 1967 : Numerical integration of a nine-level global orimitive equations model formulated by the box method. Mon. Wea. Rev., 95, 500-530.

Leith, C. E., 1968: Diffusion approximation for two-dimensional turbulence. Pliys. Fluids, 11, 671-672.

--., 1971: Atmospheric predictability and two-dinensional turbulence. $J$. Atmos. Sci.. 28, 14,5-161.

Lilly, D. K., 1969: Numerical simulation of two-dimensional turbulence. Pliys. Fluids, Suppl. II, 12, 240-2.49.

Lorenz, E. N., 1969: The predictability of a flow which possesses many scales of motion. Tellus, 21, 289-307.

Neamtan, S. M., 1946: The motion of harmonic waves in the atmosphere. J. Weteor., 3, 53-56.

Phillips, N. A., 1954: Energy transformation and meridional circulations associated with simple baroclinic waves in a twolevel quasi-geostrophic model. Tellus, 6, 273-286.

-..-, 1963: Geostrophic motion. Rev. Geophys., 1, 123-176.

Platzman, G. W., 1960: The spectral form of the vorticity equation. J. Meteor, 17, 635-644.

Robinson, G. D., 1967: Some current projects for global meteorological observation and experiment. Quart.J. Roy. Meleor. Soc., $93,409-418$

Saltzman, B., and A. Fleisher, 1962 : Spectral statistics of the wind at $500 \mathrm{mb}$. J. Atmos. Sci., 19, 195-204.

Simons, T. J., 1970: The nonlinear dynamics of cyclone waves. Ph.D. thesis, Colorado State University (Atmos. Sci. Paper No. 162$), 139 \mathrm{pp}$

Smagorinsky, J., 1969: Problems and promises of deterministic extended range forecasting. Bull. Amer. Melcor. Soc., 50 286-311.

Steers, J. A., 1965: Introduction to the Study of Map Analysis. University of London Press, 292 1m?

Wiin-Nielsen, A., 1967: On the annual variation and spectral distribution of atmospheric energy. Tellus, 19, 540-5.59. 